\title{
Plasmonic heating of gold nanoparticles and its exploitation
}

\author{
Michael Cortie ${ }^{*}$, Xiaoda Xu, Humayer Chowdhury, Hadi Zareie and Geoffrey Smith \\ Institute for Nanoscale Technology, University of Technology Sydney, \\ PO Box 123, Broadway NSW 2007, Australia
}

Keywords: Plasmon resonance, gold nanoparticle, nanorod, solar glazing

\begin{abstract}
Nanoscale particles of metals such as gold can interact with light by means of a plasmon resonance, even though they are much smaller than the wavelengths of visible light. The proportions of light that are absorbed and scattered vary with wavelength. Any light that is absorbed will cause heating of the particles, and this effect may potentially be exploited for solar glazing coatings, nanoscale lithography or medical treatments. The position of maximum absorption of an isolated spherical nanoparticle is $518 \mathrm{~nm}$, but this may be significantly red-shifted by means of decreasing the symmetry to an prolate spheroid or 'nanorod', or by producing a metal 'nanoshell' on a dielectric core, or by aggregating insulated spherical particles. Absorption peaks in the vicinity of $655 \mathrm{~nm}$ for aggregated particles and $780 \mathrm{~nm}$ for prolate spheroids are demonstrated here. Absorbed energy is released as heat into the environment of the particles, and will cause a temperature rise within the particle the magnitude of which depends upon the value of the effective heat transfer coefficient between particle and environment. The latter is not known, but we show how highly localized temperature rises of some tens of Celsius might be conceivable in systems illuminated by sunlight.
\end{abstract}

\section{INTRODUCTION}

It has been known for a century that gold nanoparticles, which are much smaller than the wavelength of light, can nevertheless exhibit a dipole particle plasmon resonance with it. The effect has even been empirically exploited as a coloring agent in glass for over two thousand years ${ }^{1}$. Factors that effect the wavelength at which the maximum absorption occurs have been much studied ${ }^{2}$. Recently, use has been made of the phenomenon for diverse biomedical assays $^{3.4}$. The geometric cross-sectional area of a gold particle of $15 \mathrm{~nm}$ diameter is $\sim 1.8 \times 10^{-16} \mathrm{~m}^{2}$ but the effective crosssection for optical extinction $\left(C_{\mathrm{ex}}\right)$ of it is a function of the wavelength of incident light. When suspended in water in dilute concentration $C_{\text {ext }}$ for a $15 \mathrm{~nm}$ diameter particle peaks at about $1.8 \times 10^{-16} \mathrm{~m}^{2}$ at a wavelength of $525 \mathrm{~nm}$. It declines .0 very low values for $\lambda>600 \mathrm{~nm}$. An extinction efficiency, $Q_{\text {ext }}$ (apparent cross section/geometric cross section) is defined, which naturally also peaks near the position of the plasmon resonance. Both absorption and scattering contribute to this extinction, with $C_{\mathrm{exl}}=C_{\mathrm{abs}}+C_{\mathrm{sca}}$, and $Q_{\mathrm{ext}}=Q_{\mathrm{abs}}+Q_{\mathrm{sca}}$, where the new subscripts refer to the cross-sections and extinction coefficients for absorption and scattering respectively. Light absorbed is released in the particle as heat, which will be subsequently shed into the immediate environment of the particle by conduction and radiation. Scattered light might not necessarily be reabsorbed, and in the present work we have considered only heating from light that is directly absorbed, that is, from $C_{\text {abs. }}$.

As the diameter of a gold particle increases towards $100 \mathrm{~nm}$, the value of $Q_{\text {ext }}$ also increases, but a large part of the increase is due to scattering rather than absorption. The trends of $Q_{\text {sca }}$ and $Q_{\text {abs }}$ with particle diameter are shown in Figs. 1 and 2. calculated using the program MiePlot $t^{5}$. It can be seen that absorption efficiency is a maximum for a gold particle of about $60 \mathrm{~nm}$ diameter, and decreases thereafter. Scattering efficiencies however continue to increase up to a diameter of about $120 \mathrm{~nm}$. The ratio of light absorbed to light scattered is shown as a function of incident wavelength and particle diameter in Fig. 3, from which it can be seen that there is a steep decrease in this parameter as particle size increases. Note however, that at incident wavelengths $>600 \mathrm{~nm}$ the particle is virtually transparent, irrespective of wavelength.

*Michael.cortie@ uts.edu.au. phone +61-2-9514-2208, www.nano.uts.edu.au 


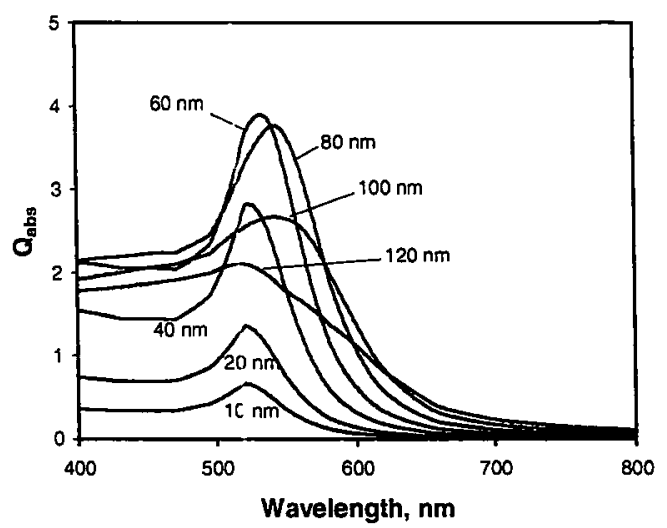

Figure 1. Calculated absorption efficiencies of gold nanoparticles suspended in water at $20^{\circ} \mathrm{C}$, as a function of wavelength of incident light and for varying diameters of particle. Calculated using the freeware program MiePlot v3.1.02 by P. Laven.

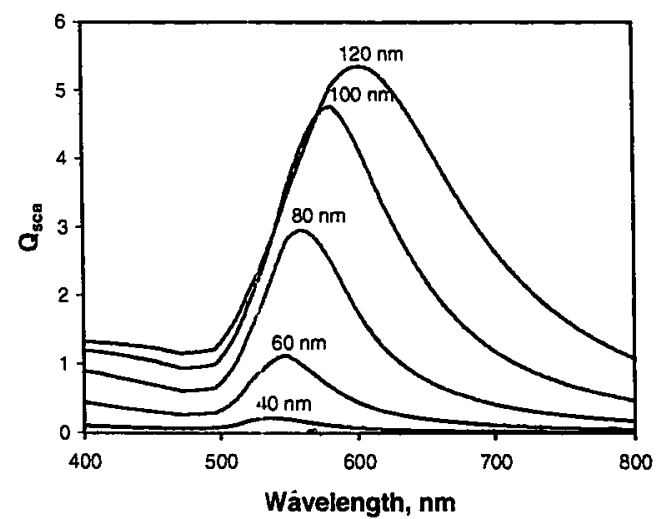

Figure 2. Calculated scattering efficiencies of gold nanoparticles suspended in water at $20^{\circ} \mathrm{C}$, as a function of wavelength of incident light and for varying diameters of particle.

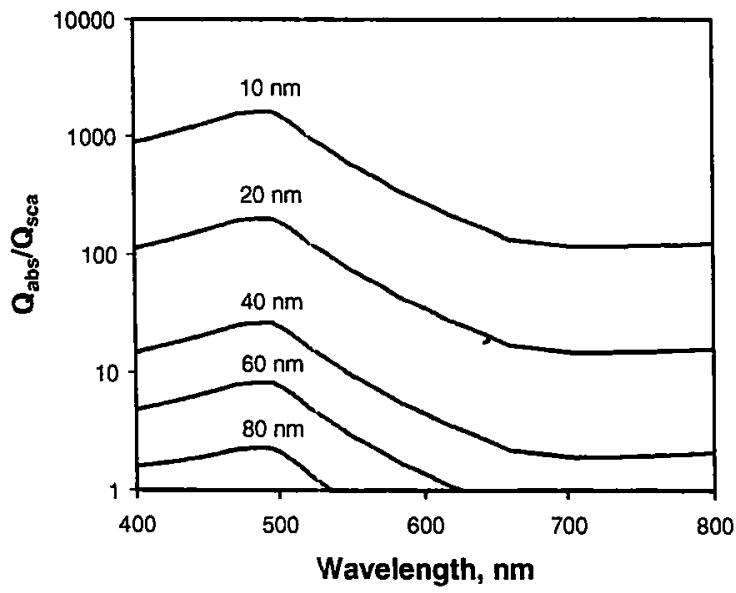

Figure 3. Ratio of $Q_{\mathrm{abs}}$ to $Q_{\mathrm{sca}}$, as a function of wavelength of incident light and diameter of gold nanoparticle.

One obvious way to exploit this effect is to deposit films of sub-60 nm gold nanoparticles onto glass windows, and to use them to block some proportion of the incident solar spectrum. The solar spectrum peaks at about $550 \mathrm{~nm}$, but an ideal solar glazing system should block incident infra-red radiation, from $750 \mathrm{~nm}$ upwards, and transmit most or all of the 
visible, from 400 to $750 \mathrm{~nm}$. The plasmon absorption of gold nanoparticles offers, at first sight, a very poor match for this. However, we will show here how the wavelength at which the plasmon absorption occurs may be red-shifted to the top of the visible spectrum by various strategies. This factor, in combination with the excellent infra-red reflectivity of gold, offers the prospect of a commercially viable process to produce gold-based solar glazing coatings by a wet chemical process.

\section{RED-SHIFTING THE PLASMON RESONANCE}

The basic plasmon resonance of a small, isolated and spherical gold particle is at a wavelength of about $518 \mathrm{~nm}$, corresponding to green light. Small changes may be made by increasing the diameter of the gold particles (as in Fig. 2) or by increasing the refractive index of the surrounding medium. However, these changes are limited to a few tens of nanometers and are insignificant compared to what can be achieved by changing the shape or state of aggregation of the particles.

The resonances of non-spherical gold particles are more complicated than those of a sphere, and all contain a component that is red-shifted with respect to the $518 \mathrm{~nm}$ peak. Two shapes are especially attractive in this regard: a thin coating of gold on a spherical dielectric core, often referred to as a 'nano-shell' ${ }^{6}$, or a cylinder or prolate spheroid of gold, usually described as a "nanorod". The plasmon resonance of the gold shell may be readily tuned into the near infra-red by varying the magnitudes of its inner and outer diameters. Fig. 4, while that of the rod may be similarly shifted by varying the latter's aspect ratio, Fig. 5. Generally however, almost any decrease in particle symmetry appears suitable to achieve some kind of red-shifting ${ }^{2.8}$.



Figure 4. General trend of the symmetrically coupled dipole plasmon resonance of gold nanoshells, plotted as a function of the ratio, $r_{1} / r_{2}$, of inner and outer radii of the shell, and the order of the resonance ( $\left.a=d i p o l e, b=q u a d r u p o l e, c=o c t u p o l e\right)$. Symbols are for diverse experimental and calculated data from Olđenburg et al. . Prodan and Nordlander $^{10}$, and Sotelo ${ }^{11}$.

There is also a third, long known but somewhat neglected, means of red-shifting the plasmon resonance. This is achieved by bringing the particles sufficiently close together that dipole-dipole interactions can occur between them ${ }^{12-16}$. This effect is negligible if $d>5 r$, where $d$ is the centre-to-centre distance and $r$ the radius of the particles, but is increasingly important at less than that ${ }^{17}$. In practice it can be most readily achieved by agglomerating spherical gold particles that are coated with an insulating dielectric since the effect will vanish if electrical contact between the particles is established. Unfortunately the phenomenon is not as readily amenable to mathematical analysis as the dipole resonance of an isolated nanoparticle, but can either be addressed by explicit numerical calculation of the absorption characteristics of a exactly defined cluster geometry ${ }^{18-20}$ or by invoking the concept of an average volume fraction of gold and a consequent 'effective dielectric constant' such as that defined by Maxwell-Garnet ${ }^{13-15}$. The peak of maximum extinction of spherical gold particles can be varied from $520 \mathrm{~nm}$ (effectively isolated particles), through to $\sim 750 \mathrm{~nm}$ (particles that are separated by only $0.5 \mathrm{~nm}$ ) by this strategy $y^{21}$, Fig 6 . Superimposed on Fig. 6 is a simple model for the effect ${ }^{15}$. The separating dielectric can be a ceramic such as $\mathrm{SiO}_{2}$, or an attached organic molecule such as citrate or mercaptoacetic acid. 
However, in practise, an increase in volume fraction cannot be used to shift the wavelength of maximum absorption to more than about $700 \mathrm{~nm}$, duie to the rapid decrease in the transparency of such dense coatings ${ }^{21}$.

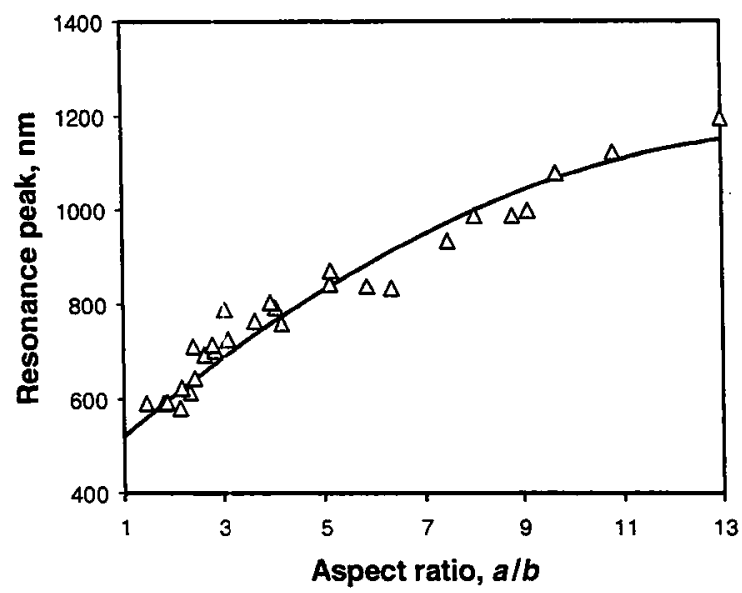

Figure 5. Position of maximum resonance of gold nanorods, for aspect ratios varying from 1:1 to 13:1. Redrawn from data of $Y u$ et al. ${ }^{7}$, with fitted regression line $\lambda=\lambda_{\text {sphere }}\left(c_{0}+c_{1}\left(\frac{a}{b}\right)+c_{2}\left(\frac{a}{b}\right)^{2}\right)$.



Figure 6. Increase in wavelength of plasmon resonance that can be obtained by aggregating gold nanoparticles. Data are from Ung $e t$ $a l^{14}$, solid line is a prediction from the theory of Genzel and Martin ${ }^{13.15}$

\section{EXPERIMENTAL}

The surface of soda-lime glass microscope slides ivas pre-treated by immersion for one hour in $5 \% \mathrm{NaOH} /$ methanol solution and then 2 minutes in $0.1 \% \mathrm{SnCl}_{2}$ in water. The slides were then rinsed with pure water before deposition of the gold from a solution of $0.0004 \mathrm{M} \mathrm{HAuCl}_{4}, 0.015 \mathrm{M} \mathrm{N}_{2} \mathrm{H}_{4}$ and $0.03 \mathrm{M}$ ethylene diamine buffered to $\mathrm{pH} 10$ with $\mathrm{NaHCO}_{3}$ $/ \mathrm{NaOH}$. This solution must be used immediately after mixing. All $\mathrm{H}_{2} \mathrm{O}$ used was purified by double-distillation. The $\mathrm{HAuCl}_{4}$ solution was prepared by dissolving pure gold in aqua regia $\left(\mathrm{HCl}: \mathrm{HNO}_{3} 3: 1 \mathrm{~V} / \mathrm{V}\right)$. Colloidal suspensions of nanorods and prolate spheroids were obtained by carrying out the reduction in the presence of hexadecyltrimethylammonium bromide $\left(\mathrm{C}_{16} \mathrm{TAB}\right)$, a surfactant. 
A Cary 5E UV/Vis/IR spectrophotometer, working in the range of $170 \mathrm{~nm}$ to $4300 \mathrm{~nm}$ and with a resolution of $0.5 \mathrm{~nm}$, was used to inspect the visible-IR transmission spectrum of the samples. The scanning rate was $5 \mathrm{~nm} / \mathrm{s}$ with spectral bandwidth (SBW) of $2 \mathrm{~nm}$. A description of the method, and additional details, may be found elsewhere ${ }^{15}$. Heat generated in the coatings may be determined by irradiation with a source of known power density and spectral characteristics, as more fully described elsewhere ${ }^{22}$.

\section{RESULTS}

The transmission spectra of two coatings of gold hemispheres on glass, both with an areal coverage of $\sim 37 \%$, are shown in Figure 7. Coating $\boldsymbol{a}$ is made up of aggregations of particles of $\sim 21 \mathrm{~nm}$ radius whereas coating $\boldsymbol{b}$ is made up of isolated nanoparticles of $58 \mathrm{~nm}$ radius. The position of maximum absorption of $\boldsymbol{a}$ is $655 \mathrm{~nm}$ whereas that of $\boldsymbol{b}$ is at $521 \mathrm{~nm}$. The red-shifting effect in $\boldsymbol{a}$ is due primarily to dipole-dipole interactions between the particles in the clusters. The clusters producing this spectrum are shown in close-up in Figure 8. The agglomerated gold particles çan be engineered to have transmission spectra with minima from $520 \mathrm{~nm}$ to as high as $750 \mathrm{~nm}^{15}$ and are arguably easier to deposit than rods or shells.

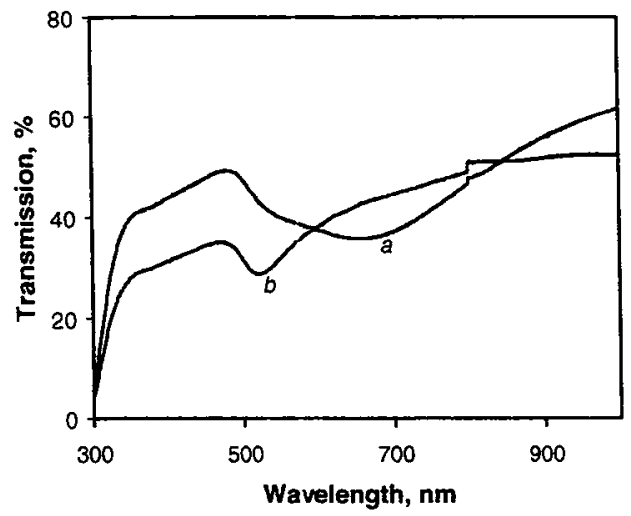

Figure 7 Transmission spectrum of glass coated with agglomerated gold nanoparticles $a$, compared to one of glass coated at a similar coverage with isolated nanoparticles, $b$.


Figure 8. Morphology of gold aggregates, a. imaged under SEM, b, with particle outlines added to guide the eye.

However, a more significant red-shift was achieved with gold nano-rods. This is shown in Figure 9, in which the absorption spectra of colloidal suspensions of gold nano-spheres and rods are compared. 


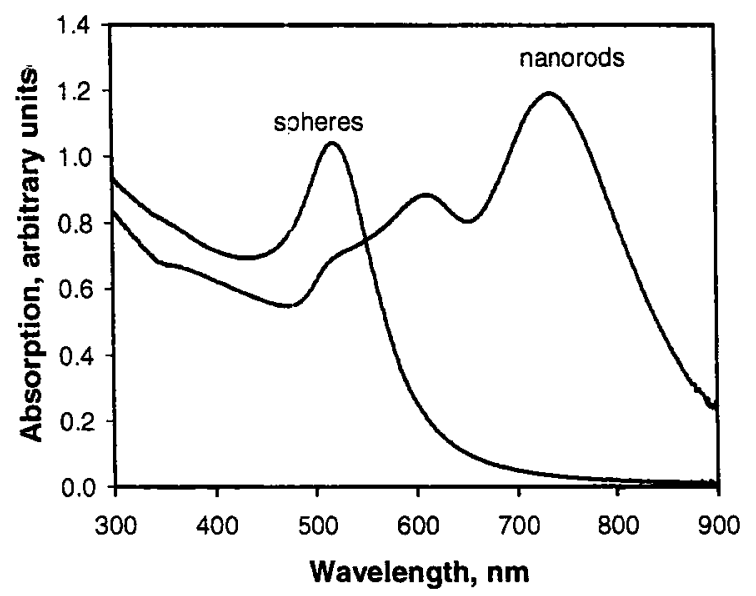

Figure 9. Comparison of the absorption spectra of gold nano-spheres and -rods, as prepared in the course of the present work.

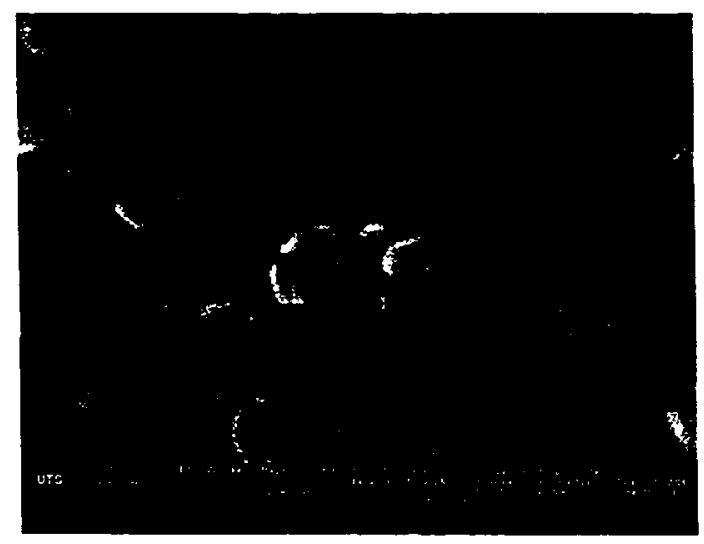

Figure 10. Prolate gold spheroids, with an aspect ratio of $\sim 2: 1$. The plasmon resonance of these is approximately $650 \mathrm{~nm}$.

\section{DISCUSSION}

\subsection{Localised heating}

The presence of the characteristic plasmon resonance on the transmission spectra of these types of coatings indicates that much of the absorption takes place by a plasmonic effect, which will be localized on the particles. Similar coatings were found in previous work to absorb between 25 and $36 \%$ of tungșten lamp radiation (Illuminant A of standard ISO 10526:1999 23 , and to transmit 60 to $65 \% \%^{22}$. The balance is reflected or scattered. The absorbed energy caused the temperature of the front face of coated glass to rise to $39^{\circ} \mathrm{C}$ under the particular still air test conditions used, at which point thermal equilibrium was established, with the absorbed heat on the surface of glass being shed into the external and internal environment of the glass by convection. Under still air conditions the coated glass sheds about half of this heat into the indoor environment. However, should the exterior air be in motion then heat transfer to the outdoor environment is enhanced, with a corresponding beneficial decrease in heat transferred indoors. It is interesting to estimate the localized temperature rise on the particles, not only because if it is sufficiently high then they might sinter and coalesce, destroying the desired plasmonic effect, but also because it might induce damage to any polymer matrix in which the gold particles might be immersed in future development of this work.

The energy absorbed by an individual isolated gold particle, can be found from 


$$
Q=\int_{0}^{\infty} C_{a b s} E_{\lambda} \cdot d \lambda
$$

where $Q$ is the power dissipated in the nanoparticle in $\mathrm{W}, C_{\mathrm{abs}}$ the extinction cross-section as $f(\lambda)$, and $E_{i}$ the spectral irradiance of the light source, W. $\mathrm{m}^{-2} \cdot \mathrm{nm}^{-1}$.

For a particle of $40 \mathrm{~nm}$ diameter exposed to a flux of sunlight at $800 \mathrm{~W} / \mathrm{m}^{2}, Q$ is $4.7 \times 10^{-13} \mathrm{~W}$. The steady-state temperature reached by the particle will depend of course on the magnitude of the heat transfer coefficient, $h$, between it and its immediate environment from

$$
Q=h . A .\left(T_{\text {paricle }}-T_{\text {environment }}\right)
$$

This expression neglects re-radiation of the,absorbed energy, which however will not be large at the comparatively low temperatures applicable here. Unfortunately, the situation on the gold coated glass panes is complicated by the fact that the particle lies at the interface between air $(k=0.026 \mathrm{~W} / \mathrm{m} / \mathrm{K})$ and glass $(k \approx 1.4 \mathrm{~W} / \mathrm{m} / \mathrm{K})$. Note also that the scale of this problem is orders smaller than the characteristic thickness of typical thermo-fluid boundary layers. Since the particles on the glass are known to be hemispheres ${ }^{15}$, and heat transfer through the air will be very much smaller in magnitude than through the glass substrate, $A$ may be taken as $\pi r^{2}$. $Q$ is known from the calculation above, and $T_{\text {environment }}$ has been measured to be $39^{\circ} \mathrm{C}^{22}$, allowing an estimate for $\mathrm{T}_{\text {particle }}$ as a function of assumed value of $h$, Figure 11. Unfortunately, the value of $h$ is not known at this time. Nevertheless, it can be seen that localized heating of the particle is certainly possible, and if this heat is not efficiently conducted away the particle temperature could rise significantly.



Figure 11. Temperature of a gold nanoparticle illuminated with $800 \mathrm{~W} / \mathrm{m}^{2}$ of sunlight. as a function of assumed value of heat transfer coefficient, $h$.

\subsection{Other applications}

Plasmonic heating could also conceivably be exploited for applications other than solar glazing. Intense localized heating around a nanoparticle can be achieved by irradiating it with a laser of the appropriate wavelength, and the resulting highly focused 'hot-spot' could be exploited in a nanolithography system. For example, a particle with $Q_{\text {abs }}$ of $\sim 4$ or 5 could be used to capture and deliver a thermal load to an underlying self-assembled monolayer, or perhaps to a surrounding liquid monomer ${ }^{24.25}$, which would be locally polymerized. Naturally, the resolution and usefulness of the system would depend in part on the ability to position gold nanoparticles in desired locations. The concept is extendible in principle to a scheme in which the gold nanoparticle or rod is attached to the tip of an atomic force microscope. In this case selected regions of the SAM can be altered by the process of positioning the nanoparticle, using the normal control electronics, while simultaneously illuminating with the laser. An arbitrary pattern of dots and lines of $\sim 5 \mathrm{~nm}$ resolution 
could be written onto the SAM. A further implication is that an array of gold nanoparticles on a suitable SAM can be conceptually be used as a write-one/read-many memory, using a near field scanning optical microscope (NSOM) to write a bit, and a scanning tunneling microscope (STS) head to read it. In principle, data storage densities in excess of those achievable by optical lithography are conceivable.

Alternatively, gold nanoparticles have been proposed as a means to deliver highly localized thermal or chemical doses onto a selected live cell type, such as a tumor cell, in a living organism. The concept is being explored by a few groups, including our own. The general idea is to coat the surface of the nanoparticle with an antibody or other molecule that will selectively attach to the target cell type, inject it into the body, and then allow it to bind on the target site. Once there the destructive effect is activated by irradiation with a wavelength at which plasmonic heating occurs. In the case of a direct targeting mechanism the destructive effect may be the heat itself ${ }^{26}$. Of course, it is not the heating from single gold nanoparticles that achieves this effect, but rather that from large numbers of particles which have been conveniently concenträted in the vicinity of the target cells. Indirect schemes, in which the effect of the irradiation is to burst a hollow nanoparticle and release some pharmaceutical compound, may also be envisaged ${ }^{27}$.

\section{CONCLUSIONS}

Nanoparticles of gold, and coatings comprised of them, can be engineered to absorb light at wavelengths between 518 $\mathrm{nm}$ and the near infra red. This may be achieved by producing particle geometries such as shells or rods using special synthesis techniques, or simply by aggregating particles coated with a dielectric layer into clusters. The energy directly absorbed by the gold particles will manifest as heat, which will be shed into its surrounding environment. The temperature rise in the immediate vicinity of the particles will be a function of the thermal conductivity of the surrounding medium and the intensity of the illumination, and could be several ${ }^{\circ} \mathrm{C}$ above that of the matrix, under appropriate conditions. This effect has possible applications in.solar glazing coatings, in nanoscale lithography, and for potential medical treatments.

\section{ACKNOWLEDGEMENT}

The support of resources and mining company AngloGold Ashanti is gratefully acknowledged.

\section{REFERENCES}

1. F.E. Wagner, S. Haslbeck, I. Stievano, S. Calogero, Q.A. Pankhurst and K.P. Martinek, "Before striking gold in goldruby glass", Nature, 407, 691-692, 2000.

2. K.L. Kelly, E. Coronado, L.L. Zhao and G.C. Schatz, "The optical properties of metal nanoparticles: the influence of size, shape, and dielectric environment", J. Phys. Chem. B, 107, 668-677, 2003.

3. M. Daniel and D. Astruc, "Gold nanoparticles: assembly, supramolecular chemistry, quantum-size-related properties, and applications toward biology, catalysis, and nanotechnology",Chem. Rev., 104, 293-346, 2004.

4. M. Bendayan, "Worth its weight in gold", Science, 291, 1363-1365.

5. P. Laven, MiePlot, available at www.philiplaven.com, accessed September 2004.

6. S.J. Oldenburg, R.D. Averitt, S.L. Westcott, N.J. Halas "Nanoengineering of optical resonances", Chemical Physics Letters, 288, 243-247, 1998.

7. Y. Yu, S. Chang, C. Lee, and C.R.C Wang, "Gold nanorods: electrochemical synthesis and optical properties",

J. Physical Chem. B, 101(34), 6661-6664, 1997.

8. N. Malikova, I. Pastoriza-Santos, M. Schierhorn, N.A. Kotov and L.M. Liz-Marzán, "Layer-by-layer assembled mixed spherical and planar gold nanoparticles: control of interparticle interactions", Langmuir, 18, 3694-3697, 2002.

9. S. J. Oldenburg, J. B. Jackson, S. L. Westcott, and N. J. Halas, "Infrared extinction properties of gold nanoshells", Applied Physics Letters, 75(19), 2897-2899, 1999.

10. E. Prodan and P. Nordlander, "Structural tunability of the plasmon resonances in metallic nanoshells", NanoLetters, 3(4), 543-547, 2003. 
11. H. Sotelo, University of Technology Sydney, private communication

12. J. Turkevich, "Colloidal gold. Part I", Gold Bulletin, 18(3), 86-91, 1985.

13. L. Genzel and T.P. Martin, "Infrared absorption by surface phonons and surface plasmons in small crystals", Surface Science, 34, 33-49, 1973.

14. T. Ung, L.M. Liz-Marzán and P. Mulvaney, "Gold nanoparticle thin films", Colloids and Surfaces A: Physicochemical and Engineering Aspects, 202, 119-126, 2002.

15. X. Xu, M. Stevens and M.B. Cortie, "In situ precipitation of gold nanoparticles onto glass for potential architectural applications", Chemistry of Materials, 16(1), 2259-2266, 2004.

16. S.L. Westcott, S.J. Oldenburg, T.R. Lee and N.J. Halas, "Construction of simple gold nanoparticle aggregates with controlled plasmon-plasmon interactions", Chemical Physics Letters, 300, 651-655, 1999.

17. J.M. Gerárdy and M. Ausloos, "Absorption spectrum of clusters of spheres from the general solution of Maxwell's equations. IV. Proximity, bulk, surface and shadow effects (in binary clusters)", Physical Review B, 27(10), 6446$6463,1983$.

18. M. Quinten and U. Kreibig, "Optical properties of aggregates of small metal particles",-Surface Science, 172, 557$577,1986$.

19. P. Etchegoin, L.F. Cohen, H. Hartigan, R.J.C. Brown, M.J.T. Milton and J.C. Gallop, "Localized plasmon resonances in inhomogeneous metallic nanoclusters", Chemical Physics Letters, 383, 577-583, 2004.

20. G.B. Smith and V.N. Pustovit, "Coupled multipolar interactions in clusters of nanoparticles with metal shells", Optics Communications, 211, 197-204, 2002.

21. T. Ung, L.M. Liz-Marzán and P. Mulvaney, "Optical properties of thin films of $\mathrm{Au}_{\mathrm{S}} @ \mathrm{SiO}_{2}$ particles",

J. Phys. Chem. B, 105, 3441-3452, 2001.

22. H. Chowdhury, X. Xu, P. Huynh and M.B. Cortie, "Radiative heat transfer across glass coated with gold nanoparticles", ASME Journal of Solar Energy Engineering, in press.

23. ISO 10526:1999 CIE Standard Illuminants for Colorimetry, International Organization for Standardization, Geneva, Switzerland.

24. B.J. Postnikova, J. Currie, T. Doyle, R.E. Hanes, E.A. Anslyn, J.B. Shear, D.E. Vanden Bout, Towards nanoscale three-dimensional fabrication using two-photon initiated polymerization and near-field excitation, Microelectronic Engineering 69, 459-465, 2003.

25. F. Caciallia, R. Riehn, A. Downes, G. Latini, A. Charas, J.Morgado, "Fabrication of conjugated polymers nanostructures via direct near-field optical lithography", Ultramicroscopy, 100, 449-455, 2004.

26. D.P. O'Neal, L.R. Hirsch, N.J. Halas, J.D. Payne and J.L. West, "Photo-thermal tumor ablation in mice using near infrared-absorbing nanoparticles", Cancer Letters, 2(19(2),171-176, 2004.

27. B. Radt, T.A. Smith, F. Caruso, Advanced Materials, 2004, in press. 


\section{PROCEEDINGS OF SPIE}

\section{Smart Structures, Devices, and Systems II}

Said F. Al-Sarawi

Chair/Editor

13-15 December 2004

Sydney, Australia

Sponsored by

SPIE-The International Society for Optical Engineering

Cosponsored by

University of New South Wales (Australia)

Australian Government Department of Defence,

Defence Science and Technology Organisation

Cochlear (Australia)

MSMR-NSW Ministry for Science and Medical Research (Australia)

Cooperating Organizations

The University of Adelaide (Australia)

Centre for Biomedical Engineering, Adelaide (Australia) 


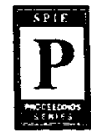

The papersincluded in this volume were part of the technical conference cited on the cover and title page. Papers were selected and subject to review by the editors and conference program committee Some conference presentations may not be available for publication. The papers published in these proceedings reflect the work and thoughts of the authors and are published herein as submitted. The publisher is not responsible for the validity of the information or for any outcomes resulting from reliance thereon.

Please use the following format to cite material from this book:

Author(s), 'Title of Paper," in Smart Structures, Devices, and Systems II, edited by Said F. Al-Sarawi, Proceedings of SPIE Vol. 5649 (SPIE, Bellingham, WA, 2005) page numbers.

ISSN 0277-786X

ISBN 0-8194-5609-8

Published by

SPIE-The International Society for Optical Engineering

P.O. Box 10, Bellingham, Washington 98227-0010 USA

Telephone 1 360/676-3290 (Pacific Time) - Fax 1 360/647-1445

http://mww.spie.org

Copyright (C) 2005, The Society of Photo-Optical Instrumentation Engineers

Copying of material in this book for internal or personal use, or for the internal or personal use of specific clients, beyond the fair use provisions granted by the U.S. Copyright Low is authorized by SPIE subject to payment of copying fees. The Transactional Reporting Service base fee for this volume is $\$ 15.00$ per article (or portion thereof), which should be paid directly to the Copyright Clearance Center (CCC), 222 Rosewood Drive, Danvers, MA 01923. Payment may also be made electronically through $\mathrm{CCC}$ Online at http://mww.copyright.com. Other copying for republication, resale, advertising or promotion, or any form of systematic or multiple reproduction of any material in this book is prohibited except with permission in writing from the publisher. The CCC fee code is 0277 . $786 \times 105 / \$ 15.00$.

Printed in the United States of America. 